\title{
Soft Robotics for the Hydraulic Atlas Arms: Joint Impedance Control with Collision Detection and Disturbance Compensation
}

\author{
Jonathan Vorndamme, Moritz Schappler, Alexander Tödtheide and Sami Haddadin ${ }^{1}$.
}

\begin{abstract}
Soft robotics methods such as impedance control and reflexive collision handling have proven to be a valuable tool to robots acting in partially unknown and potentially unstructured environments. Mainly, the schemes were developed with focus on classical electromechanically driven, torque controlled robots. There, joint friction, mostly coming from high gearing, is typically decoupled from link-side control via suitable rigid or elastic joint torque feedback. Extending and applying these algorithms to stiff hydraulically actuated robots poses problems regarding the strong influence of friction on joint torque estimation from pressure sensing, i.e. link-side friction is typically significantly higher than in electromechanical soft robots. In order to improve the performance of such systems, we apply state-of-the-art fault detection and estimation methods together with observer-based disturbance compensation control to the humanoid robot Atlas. With this it is possible to achieve higher tracking accuracy despite facing significant modeling errors. Compliant end-effector behavior can also be ensured by including an additional force/torque sensor into the generalized momentum-based disturbance observer algorithm from [1].
\end{abstract}

\section{Introduction AND StATE of THE ART}

Compliant manipulation and appropriate reflex reactions to collisions have been an active research field over the last decades, opening the door to safer and more autonomous robot applications [2], [3], [4]. Human-friendly robot behavior has to be ensured not only for industrial robotic coworkers, which are typically serial chain manipulators, but also in future healthcare, rescue or even personal robotics applications, where mobility is essential. At the same time, compliance control and reflexive contact handling are sought to be essential features in damage protection for systems as the Boston Dynamics Atlas hydraulic robot [5], [6].

Essentially, softness is either achieved by an inherently compliant structure [7], [8] and/or active compliance control via high-fidelity joint torque feedback. One of the most prominent control concepts to implement compliance is impedance control. It was introduced in [9] and extended to flexible joint robots e.g. in [10], [11]. However, up to now the schemes were mainly applied to electromechanically driven robots. For hydraulic humanoid robots, basic compliance control schemes were implemented on the SARCOS

\footnotetext{
1 All authors are with Institute of Automatic Control (IRT), Leibniz Universität Hannover, Appelstr. 11, 30167 Hannover, Germany.

Email: <lastname>@irt.uni-hannover.de.

This work is part of a project that has received funding from the European Union's Horizon 2020 research and innovation programme under grant agreement No 688857 ("SoftPro").

The authors would like to thank the other members of Team ViGIR for supporting the robot experiments. The robot experiments were supported in by the Defense Advanced Research Projects Agency (DARPA) under Air Force Research Laboratory (AFRL) contract FA8750-12-C-0337.
}

humanoid, focussing on balancing and contact Jacobians [12], on constraint handling via quadratic programming [13] or with LQR feedback gain optimization [14]. The concept of admittance control (position-based impedance control) has been implemented on the Atlas robot in [15].

Activating reflex reactions as a response to potentially unwanted environmental contacts is another main pillar in safe and sensitive robot interaction. This requires the ability to discriminate internal from external torques based on accurate dynamics models together with proprioceptive position and torque measurements. Disturbance observers are a common technique in robotics to handle either modeling inaccuracies [16], [17] or to recognize unexpected events such as collisions. [18] used a momentum-based disturbance observer for collision detection, isolation, and estimation, including validation with a 2-Degree-of-Freedom (DoF) simulation. These results were extended to the flexible manipulator case and experimentally validated with the DLR lightweight robot arm III [1], using the concepts of total link energy and generalized momentum. An analysis of different terms in the error dynamics and an approach for velocity-based variable collision thresholds were presented in [19]. An estimation of the external end-effector wrenches based on observed disturbance torques was used in [20] to enhance a model predictive balancing controller on the humanoid robot TORO.

In [21] the subsequent collision classification problem was approached by applying state-of-the-art machine learning techniques to learning the collision torque profiles of different collision types, including features based on collision frequencies, amplitudes or other physically motivated aspects. A summary of collision handling can be found in [2]. The focus of these works was on electromechanically actuated robots equipped with link-side joint torque sensing.

A collision detection for the 6-DoF hydraulic robot arm Maestro with low-pass filtered model error was evaluated in [22], focussing on hydraulic friction effects. In [23] the authors applied a disturbance observer for collision detection that contains a bandpass filter, making use of specifically identified collision frequencies, to a 3-DoF hydraulic robot arm with joint torque sensors.

In contrast to their electromechanical counterpart, and due to the fact that no high gearing with according friction losses is required, commonly used hydraulic actuators do not have link-side torque measurements. In fact, actuator forces can be estimated via the chamber pressures. However, link-side friction, e.g. induced by the chamber seals, can be rather high indeed. This in turn makes direct high-fidelity joint torque 
control, and thus also any other soft-robotics control concept, difficult to realize.

In the present work, we approach this problem by identifying friction and the required dynamic parameters for our model-based control concept with a two-step method. In the first step, a friction model including Coulomb and viscous friction is identified. In the second step, the results of the first step are used to reduce the number of parameters for the identification of the dynamics model.

In order to compensate for model errors when using impedance control, a generalized momentum-based disturbance observer is used [24]. As a drawback compliance is lost, since external forces cannot be systematically discriminated from the disturbance torques and are thus compensated by the observer as well. To overcome this problem for end-effector contacts we use a wrist force/torque sensor to calculate the external joint torques and exclude them from the disturbance torque calculation. Finally, an identified friction model is part of the control scheme to further reduce the observed disturbances.

The contributions of this paper are

1) the extension of momentum-based collision handling by considering effects such as friction and wrist force/torque measurements,

2) the experimental validation of disturbance observerenhanced collision detection and reaction schemes to the hydraulic part of the 7-DoF Atlas robot arms,

3) simulative results of a disturbance observer compensation concept based on wrist force/torque measurement increasing the impedance controller precision and keeping the end-effector compliance simultaneously, and

4) a two-step friction and extended rigid body dynamics identification method applied to a serial chain robot with both hydraulic and electromechanic actuators.

The paper is organized as follows. Section II adapts our identification and control concepts from [24] and compares the results to the previous approach. Section III shows experimental results for collision detection and reaction with the Atlas robot. Furthermore, the concept of regaining compliance when using disturbance compensation from [16] for our controller implementation is explained. Section IV concludes the paper.

\section{Modeling AND IDENTIFICATION}

In this section we shortly review the system modeling and enhanced impedance controller approach (II-A), modifications to the momentum-based disturbance observer to include wrist wrench measurements (II-B), compensation of modeling errors and collision handling (II-C), and an identification scheme that iteratively estimates friction characteristics and rigid body parameters (II-D).

\section{A. System Model and Joint Impedance Controller}

This work focuses on the 7R type serial chain arms of the Boston Dynamics humanoid robot Atlas, see Fig. 9. The arms employ a hybrid actuation concept where the first four

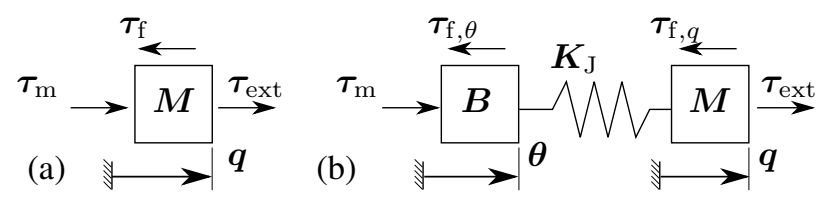

Fig. 1: Relevant torques acting along the mechanical structure for the rigid joint model (a) with link inertia $M$ and the flexible joint model (b) with motor inertia $\boldsymbol{B}$ and motor position $\boldsymbol{\theta}$.

joints (shz, shx, ely, elx) are hydraulic, and the wrist joints (wry, wrx, wry2) are driven by electromechanical gear drives. We assume the standard fixed-base rigid joint arm model

$$
\boldsymbol{M}(\boldsymbol{q}) \ddot{\boldsymbol{q}}+\boldsymbol{C}(\boldsymbol{q}, \dot{\boldsymbol{q}}) \dot{\boldsymbol{q}}+\boldsymbol{g}(\boldsymbol{q})=\boldsymbol{\tau}_{\mathrm{m}}-\tau_{\mathrm{f}}+\tau_{\mathrm{ext}}
$$

with generalized joint position $\boldsymbol{q} \in \mathbb{R}^{n_{\mathrm{j}}}$ ( $n_{\mathrm{j}}$ being the number of joints), positive definite and symmetric inertia matrix $\boldsymbol{M}(\boldsymbol{q})$, centrifugal and Coriolis matrix $\boldsymbol{C}(\boldsymbol{q}, \dot{\boldsymbol{q}})$, gravity torque vector $\boldsymbol{g}(\boldsymbol{q})$, actuator torques $\boldsymbol{\tau}_{\mathrm{m}}$, friction torques $\boldsymbol{\tau}_{\mathrm{f}}$ and external torques $\tau_{\text {ext }}$. The friction torque $\tau_{\mathrm{f}}$ is composed of the motor side friction $\tau_{\mathrm{f}, \theta}$ and the link side friction $\tau_{\mathrm{f}, q}$. Both hydraulic and electromechanic actuators are considered as ideal torque sources, generating a motor torque $\tau_{\mathrm{m}}$, allowing a separation of the actuator dynamics from the rigid body model.

For soft-robotics control of the system we chose the joint impedance control torque $\tau_{\mathrm{d}}$ to be

$$
\begin{gathered}
\boldsymbol{\tau}_{\mathrm{d}}=\boldsymbol{K}\left(\boldsymbol{q}_{\mathrm{d}}-\boldsymbol{q}\right)+\boldsymbol{D}\left(\dot{\boldsymbol{q}}_{\mathrm{d}}-\dot{\boldsymbol{q}}\right)+\hat{\boldsymbol{g}}(\boldsymbol{q}) \\
+\hat{\boldsymbol{C}}(\boldsymbol{q}, \dot{\boldsymbol{q}}) \dot{\boldsymbol{q}}+\hat{\boldsymbol{M}}\left(\boldsymbol{q}_{\mathrm{d}}\right) \ddot{\boldsymbol{q}}_{\mathrm{d}}+\kappa_{\mathrm{f}} \hat{\boldsymbol{\tau}}_{\mathrm{f}}(\dot{\boldsymbol{q}})-\kappa_{\varepsilon} \hat{\boldsymbol{\tau}}_{\varepsilon}\left(\boldsymbol{q}, \dot{\boldsymbol{q}}, \boldsymbol{\tau}_{\mathrm{m}}\right),
\end{gathered}
$$

where $\boldsymbol{q}_{\mathrm{d}}, \dot{\boldsymbol{q}}_{\mathrm{d}}, \ddot{\boldsymbol{q}}_{\mathrm{d}}$ are the desired position, velocity, and acceleration, respectively. The matrices $\boldsymbol{K}=\operatorname{diag}\left\{k_{i}\right\}$ and $\boldsymbol{D}_{\xi}=$ $\operatorname{diag}\left\{d_{\xi, i}\right\}$ denote diagonal positive definite stiffness and modal damping and $\boldsymbol{D}$ the resulting positive definite damping matrix. $\hat{g}$ and $\hat{C}$ are the gravity and centrifugal/Coriolis estimates. The inertial feedforward term makes use of the estimated inertia matrix $\hat{M}$ as a function of the desired position $\boldsymbol{q}_{\mathrm{d}}$. The compensation terms $\hat{\boldsymbol{\tau}}_{\mathrm{f}}(\dot{\boldsymbol{q}})$ (viscous and Coulomb friction) and $\hat{\boldsymbol{\tau}}_{\varepsilon}\left(\boldsymbol{q}, \dot{\boldsymbol{q}}, \boldsymbol{\tau}_{\mathrm{m}}\right)$ (estimated disturbance from Sec. II-B) are activated via the scalars $\kappa_{\mathrm{f}} \in\{0,1\}$ and $\kappa_{\varepsilon} \in\{0,1\}$, respectively.

Combining (1) and (2) leads to the closed-loop dynamics

$$
\begin{gathered}
\boldsymbol{M}(\boldsymbol{q}) \ddot{\boldsymbol{q}}-\hat{\boldsymbol{M}}\left(\boldsymbol{q}_{\mathrm{d}}\right) \ddot{\boldsymbol{q}}_{\mathrm{d}}+\boldsymbol{D}\left(\dot{\boldsymbol{q}}-\dot{\boldsymbol{q}}_{\mathrm{d}}\right)+\boldsymbol{K}\left(\boldsymbol{q}-\boldsymbol{q}_{\mathrm{d}}\right) \\
=\boldsymbol{\tau}_{\mathrm{ext}}+\boldsymbol{\delta}-\kappa_{\varepsilon} \hat{\boldsymbol{\tau}}_{\varepsilon},
\end{gathered}
$$

where $\delta$ denotes lumped dynamics and friction modeling errors and errors caused by sensor drift, offsets and time delays. We assume these effects to be additive.

\section{B. Disturbance Observer}

Before introducing our observer design, let us shortly summarize the underlying problem of collision detection with typical hydraulic robots. Figure 1 emphasizes the friction torques that are relevant for rigid joint models in comparison to the flexible joint case. The latter represents e.g. electromechanically actuated robots with elastic joints and torque sensing [11]. In the former case, the total friction $\tau_{f}$ and external torques $\tau_{\text {ext }}$ act on a single body that 


\section{This is the author's version of an article that has been published in the IROS 2016 proceedings.}

Changes were made to this version by the publisher prior to publication.

The final version of record is available at http://dx.doi.org/10.1109/iros.2016.7759517

represents both motor and link inertia and sum up to a total disturbance torque. Thus, except under certain modeling assumptions they cannot be separated with standard proprioceptive sensing and according observer techniques. In the latter case, motor and link-side dynamics are coupled via the joint stiffness $\boldsymbol{K}_{\mathrm{J}}$. Typically, this originates either from rather elastic gears such as Harmonic Drives in combination with joint torque sensors (rather high inherent stiffness $\boldsymbol{K}_{\mathrm{J}}$ ), or from deliberately placed spring elements as e.g. in the Series Elastic Actuation (SEA [7]) case (rather low inherent stiffness $\boldsymbol{K}_{\mathrm{J}}$ ). Since the link side friction $\boldsymbol{\tau}_{\mathrm{f}, q}$ is usually low (it is mainly caused by low friction link-side bearings), it may be neglected and the link-side observer essentially estimates the true external joint torques [2]. Therefore, it is possible to set up two observer schemes, one for the actuator side estimating $\boldsymbol{\tau}_{\mathrm{f}, \theta}$ [25], and one for the link side estimating $\boldsymbol{\tau}_{\text {ext }}$. Note that one can obviously set up an elastic joint model for the hydraulic case as well. However, this would only have a similar implication if an additional joint torque sensor for decoupling would be inserted after link friction.

To be able to distinguish between internal and external effects, we extend the momentum-based disturbance observer from [26], [27], [2] to include measurements of external

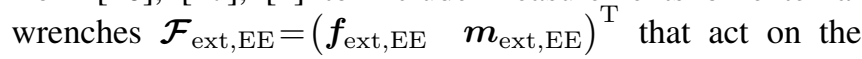
end-effector, i.e. "after" the sensor [20]. The extended residual for end-effector contacts is then defined as

$$
\hat{\boldsymbol{\tau}}_{\varepsilon}=\boldsymbol{K}_{\mathrm{o}}\left(\hat{\boldsymbol{M}}(\boldsymbol{q}) \dot{\boldsymbol{q}}-\int_{0}^{t}\left[\boldsymbol{\tau}_{\mathrm{m}}-\gamma(\boldsymbol{q}, \dot{\boldsymbol{q}})+\hat{\boldsymbol{\tau}}_{\varepsilon}-\boldsymbol{\alpha}(\boldsymbol{q}, \dot{\boldsymbol{q}})\right] \mathrm{d} \tilde{t}\right),
$$

where $\boldsymbol{K}_{\mathrm{o}}=\operatorname{diag}\left\{k_{\mathrm{o}, i}\right\}>\mathbf{0}$ is the observer gain matrix and

$$
\gamma(\boldsymbol{q}, \dot{\boldsymbol{q}}):=\hat{\boldsymbol{g}}(\boldsymbol{q})+\hat{\boldsymbol{C}}(\boldsymbol{q}, \dot{\boldsymbol{q}}) \dot{\boldsymbol{q}}-\dot{\hat{\boldsymbol{M}}}(\boldsymbol{q}) \dot{\boldsymbol{q}}=\hat{\boldsymbol{g}}(\boldsymbol{q})-\hat{\boldsymbol{C}}^{\mathrm{T}}(\boldsymbol{q}, \dot{\boldsymbol{q}}) \dot{\boldsymbol{q}} .
$$

Equality (5) follows directly from the skew-symmetry of $\dot{\hat{M}}(\boldsymbol{q})-2 \hat{\boldsymbol{C}}(\boldsymbol{q}, \dot{\boldsymbol{q}})$ [1]. The vector $\boldsymbol{\alpha}(\boldsymbol{q}, \dot{\boldsymbol{q}})$ is defined as

$$
\begin{aligned}
\boldsymbol{\alpha}(\boldsymbol{q}, \dot{\boldsymbol{q}}): & =\kappa_{\mathrm{f}} \hat{\boldsymbol{\tau}}_{\mathrm{f}}(\dot{\boldsymbol{q}})-\kappa_{\mathrm{ext}} \boldsymbol{\tau}_{\text {ext,EE }} \\
& =\kappa_{\mathrm{f}} \hat{\boldsymbol{\tau}}_{\mathrm{f}}(\dot{\boldsymbol{q}})-\kappa_{\mathrm{ext}} \boldsymbol{J}^{\mathrm{T}}(\boldsymbol{q}) \mathcal{F}_{\mathrm{ext}, \mathrm{EE}}
\end{aligned}
$$

The contact wrench $\mathcal{F}_{\text {ext,EE }}$ is typically measured with a load-compensated force/torque sensor in the robot wrist. The resulting external joint torques $\tau_{\text {ext,EE }}$ are obtained by the well known mapping via the end-effector Jacobian $\boldsymbol{J}(\boldsymbol{q})$. Its feedback is activated via $\kappa_{\text {ext }} \in\{0,1\}$. Note that the components of $\mathcal{F}_{\text {ext,EE }}$ that are in the kernel of $\boldsymbol{J}^{\mathrm{T}}(\boldsymbol{q})$ are absorbed by the structure of the robot and are not reflected in $\tau_{\text {ext,EE}}$.

To distinguish directly measurable joint torques originating from external wrenches at the end-effector $\tau_{\text {ext,EE }}$ from joint torques caused by external wrenches at the structure $\tau_{\text {ext,links }}$ that cannot be measured by the wrist sensor, we define the total external torque vector $\tau_{\text {ext }}$ to be

$$
\tau_{\text {ext }}:=\tau_{\text {ext,EE }}+\tau_{\text {ext,links }} .
$$

The true disturbance joint torque $\boldsymbol{\tau}_{\varepsilon}$ for rigid joint models consists of the joint torques from (7) plus the error term $\delta$ in (3). As derived in [2] the observed disturbance torque,

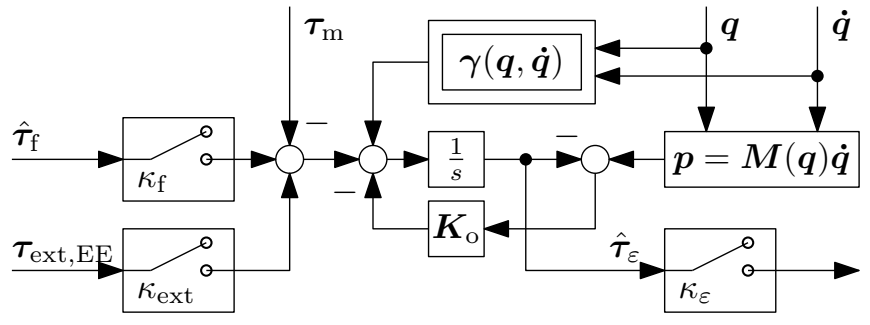

Fig. 2: Flowchart of the proposed observer structure.

also for this extended form, converges element-wise with first order dynamics (presented in frequency domain)

$$
\hat{\tau}_{\varepsilon, i}=\frac{k_{\mathrm{o}, i}}{s+k_{\mathrm{o}, i}}\left(\tau_{\text {ext }, \mathrm{EE}, i}\left(1-\kappa_{\mathrm{ext}}\right)+\tau_{\text {ext }, \text { link }, i}+\delta_{i}\right)
$$

where $1 / k_{\mathrm{o}, i}$ is the time constant. Figure 2 depicts an overview of the overall observer structure.

In the next section, we outline how this extended disturbance observer is used to compensate for model errors and detect collisions simultaneously.

\section{Compensation of Model Errors and Collision Detection}

Assuming $\tau_{\text {ext }}$ to have slower dynamics than the observer (sufficiently large observer gain $\boldsymbol{K}_{\mathrm{o}}$ ). One can approximate the disturbance torque as $\hat{\boldsymbol{\tau}}_{\varepsilon} \approx\left(\boldsymbol{\tau}_{\mathrm{ext}, \mathrm{EE}}\left(1-\kappa_{\mathrm{ext}}\right)+\boldsymbol{\tau}_{\mathrm{ext}, \mathrm{link}}+\boldsymbol{\delta}\right)$. Therefore, one obtains from equation (3)

$$
\begin{aligned}
& \boldsymbol{M}(\boldsymbol{q}) \ddot{\boldsymbol{q}}-\hat{\boldsymbol{M}}\left(\boldsymbol{q}_{\mathrm{d}}\right) \ddot{\boldsymbol{q}}_{\mathrm{d}}+\boldsymbol{D}\left(\dot{\boldsymbol{q}}-\dot{\boldsymbol{q}}_{\mathrm{d}}\right)+\boldsymbol{K}\left(\boldsymbol{q}-\boldsymbol{q}_{\mathrm{d}}\right) \\
= & \boldsymbol{\tau}_{\text {ext }}+\boldsymbol{\delta}-\kappa_{\varepsilon}\left(\boldsymbol{\tau}_{\text {ext,EE }}\left(1-\kappa_{\text {ext }}\right)+\boldsymbol{\tau}_{\text {ext,link }}+\boldsymbol{\delta}\right) .
\end{aligned}
$$

The trajectory tracking performance can thus be improved significantly by using the disturbance compensation with $\kappa_{\varepsilon}=1$ in (2) and $\kappa_{\text {ext }}=0$ in (6), as this would eliminate model inaccuracies $\delta$. The obvious drawback would be the loss of compliance w.r.t. external torques, since equation (9) becomes

$$
\boldsymbol{M}(\boldsymbol{q}) \ddot{\boldsymbol{q}}-\hat{\boldsymbol{M}}\left(\boldsymbol{q}_{\mathrm{d}}\right) \ddot{\boldsymbol{q}}_{\mathrm{d}}+\boldsymbol{D}\left(\dot{\boldsymbol{q}}-\dot{\boldsymbol{q}}_{\mathrm{d}}\right)+\boldsymbol{K}\left(\boldsymbol{q}-\boldsymbol{q}_{\mathrm{d}}\right)=\mathbf{0} .
$$

Thus, the system no longer reacts to external forces in case of precise disturbance estimates. This unwanted increase in stiffness could be avoided for interaction with the endeffector by exploiting wrist wrench sensing. Setting $\kappa_{\varepsilon}=1$ in (2) and $\kappa_{\text {ext }}=1$ in (6), the closed loop behavior (3) becomes

$\boldsymbol{M}(\boldsymbol{q}) \ddot{\boldsymbol{q}}-\hat{\boldsymbol{M}}\left(\boldsymbol{q}_{\mathrm{d}}\right) \ddot{\boldsymbol{q}}_{\mathrm{d}}+\boldsymbol{D}\left(\dot{\boldsymbol{q}}-\dot{\boldsymbol{q}}_{\mathrm{d}}\right)+\boldsymbol{K}\left(\boldsymbol{q}-\boldsymbol{q}_{\mathrm{d}}\right)=\boldsymbol{\tau}_{\mathrm{ext}, \mathrm{EE}}$.

This scheme has similarities to the one proposed in [16] and will be termed disturbance compensation (DC) with external forces compliance (EFC) from now on. A qualitative comparison for the different settings of $\kappa_{\varepsilon}$ and $\kappa_{\text {ext }}$ is shown in the Table of Fig. 3.

In summary, it is possible to

1) detect end-effector contacts with the inertia compensated wrench sensor,

2) detect contacts along the entire robot structure beyond a tolerance band with the extended observer, 


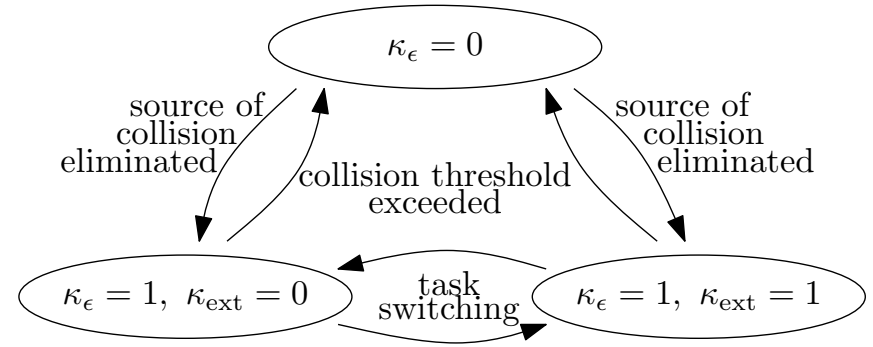

\begin{tabular}{|l|c|c|c|}
\hline Property & $\kappa_{\varepsilon}=0$ & $\kappa_{\varepsilon}=1, \kappa_{\text {ext }}=0$ & $\kappa_{\varepsilon}=\kappa_{\text {ext }}=1$ \\
\hline Increased accuracy & $\times$ & $\checkmark$ & $\checkmark$ \\
\hline End-effector compliance & $\checkmark$ & $\times$ & $\checkmark$ \\
\hline Link compliance & $\checkmark$ & $\times$ & $\times$ \\
\hline Closed-loop behavior & $(3)$ & $(10)$ & $(11)$ \\
\hline
\end{tabular}

Fig. 3: Context sensitive control and reaction scheme and comparison between different setups of controller (2). Control mode $\kappa_{\text {ext }}=1$ is used for tasks which need end-effector compliance, $\kappa_{\text {ext }}=0$ for tasks which do not. When a collision is detected, the controller switches into compliant mode $\left(\kappa_{\varepsilon}=0\right)$.

3) render end-effector compliance while having stiff behavior for contacts along the robot structure.

The missing compliance for link collisions can still be encountered by switching to compliance control as soon as a link collision is detected.

Using the above observer, we implemented a simple collision detection scheme that is based on a constant disturbance joint torque threshold $\zeta$.

$$
\text { CollDet }= \begin{cases}1, & \text { if } \hat{\tau}_{\varepsilon}>\zeta(\text { component wise) } \\ 0, & \text { otherwise }\end{cases}
$$

For (12) to work properly, one has to solve the trade-off between robustness and convergence speed of the observer. The first order observer dynamics in (8) with time constant $1 / k_{\mathrm{o}, i}$ makes the collision detection robust against sensor noise and peaks, as long as $\boldsymbol{K}_{\mathrm{o}}$ is not chosen too large. However, large $\boldsymbol{K}_{\mathrm{o}}$ leads to faster convergence of $\hat{\boldsymbol{\tau}}_{\varepsilon}$ to $\boldsymbol{\tau}_{\text {ext }}$.

For collision reaction, in this work, we switch to gravity compensation mode [2]:

$$
\boldsymbol{\tau}_{\mathrm{d}}= \begin{cases}\hat{\boldsymbol{g}}(\boldsymbol{q}), & \text { if CollDet }=1 \\ \boldsymbol{\tau}_{\mathrm{d}} \text { from (2), } & \text { otherwise }\end{cases}
$$

Another possibility would be to implement the scheme depicted in Fig. 3. It uses the three control modes in the table in a context sensitive manner. For example, for grasping objects end-effector compliance and high position accuracy is needed, therefore $\kappa_{\varepsilon}=1$ and $\kappa_{\text {ext }}=1$ is used. For moving objects of unknown weight, end-effector compliance is not wanted and therefore $\kappa_{\text {ext }}=1$ is used. Finally, if the collision threshold is exceeded, the robot switches into full compliant mode $\left(\kappa_{\varepsilon}=0\right)$ to avoid damage.

Next, we outline our system identification and friction modeling approach.

\section{Rigid Body and Friction Identification}

In our previous work [24] both, rigid body model and friction were simultaneously identified as the parameter vector $\boldsymbol{\beta}_{\mathrm{I}}$. The results still contained a relevant error regarding model fitting and plausibility of the friction parameters compared to single-joint experiments. Therefore, in this work a sequential procedure was applied, where pre-identified Coulomb and viscous friction parameters $\boldsymbol{d}_{\mathrm{v}, \mathrm{p}}, \boldsymbol{\mu}_{\mathrm{C}, \mathrm{p}}$ were included into the identification procedure in order to reduce the parameter space from 59 to 45 unknowns $^{1}$.

1) Identification Model and Approach: For identification purposes the robot dynamics (1), including a suitable friction model, can be written in regressor form as

$$
\boldsymbol{\tau}_{\mathrm{m}}=\boldsymbol{\Phi} \boldsymbol{\beta}-\boldsymbol{\tau}_{\mathrm{ext}}
$$

where the regressor matrix $\boldsymbol{\Phi}$ contains distinct base and friction parameter related columns $\boldsymbol{\Phi}=\left(\begin{array}{ll}\boldsymbol{\Phi}_{\mathrm{b}} & \boldsymbol{\Phi}_{\mathrm{f}}\end{array}\right)$. The elements of the parameter vector $\boldsymbol{\beta}=\left(\boldsymbol{\beta}_{\mathrm{b}} \boldsymbol{d}_{\mathrm{v}} \boldsymbol{\mu}_{\mathrm{c}}\right)^{\mathrm{T}}$ denote the base, viscous friction and Coulomb friction parameter vectors. The regressor matrix of the friction model can be allocated as

$$
\boldsymbol{\Phi}_{\mathrm{f}}(\dot{\boldsymbol{q}})=(\operatorname{diag}\{\dot{\boldsymbol{q}}\} \quad \operatorname{diag}\{\operatorname{sgn}(\dot{\boldsymbol{q}})\})
$$

Assuming $\tau_{\text {ext }}=\mathbf{0}$ during identification procedure, the influence of (15) can be incorporated by subtracting (15) from both sides in (14). This leads to the friction-corrected motor torque

$$
\begin{aligned}
\boldsymbol{\tau}_{\mathrm{m}, \mathrm{f}} & =\boldsymbol{\tau}_{\mathrm{m}}-\boldsymbol{\Phi}_{\mathrm{f}}(\dot{\boldsymbol{q}})\left(\boldsymbol{d}_{\mathrm{v}}, \boldsymbol{\mu}_{\mathrm{c}}\right)^{\mathrm{T}} \\
& =\boldsymbol{\Phi} \boldsymbol{\beta}-\boldsymbol{\Phi}_{\mathrm{f}}(\dot{\boldsymbol{q}})\left(\boldsymbol{d}_{\mathrm{v}}, \boldsymbol{\mu}_{\mathrm{c}}\right)^{\mathrm{T}}=\boldsymbol{\Phi}_{\mathrm{b}} \boldsymbol{\beta}_{\mathrm{b}}
\end{aligned}
$$

Identifying the numerical values of $\boldsymbol{\beta}_{\mathrm{b}}$ is done using a Moore-Penrose pseudoinverse

$$
\hat{\boldsymbol{\beta}}_{\mathrm{b}}=\left(\boldsymbol{F}^{\mathrm{T}} \boldsymbol{\Sigma}^{-1} \boldsymbol{F}\right)^{-1} \boldsymbol{F}^{\mathrm{T}} \boldsymbol{\Sigma}^{-1} \boldsymbol{b}
$$

filled with experimentally gained optimized Fourier-based joint angle trajectories of duration $t_{\mathrm{f}}$ [28]. The information matrix $\boldsymbol{F}$ and the measurement vector $\boldsymbol{b}$ are defined as

$$
\boldsymbol{F}=\left(\begin{array}{c}
\boldsymbol{\Phi}_{\mathrm{b}}\left(\boldsymbol{q}\left(t_{1}\right), \dot{\boldsymbol{q}}\left(t_{1}\right), \ddot{\boldsymbol{q}}\left(t_{1}\right)\right) \\
\boldsymbol{\Phi}_{\mathrm{b}}\left(\boldsymbol{q}\left(t_{2}\right), \dot{\boldsymbol{q}}\left(t_{2}\right), \ddot{\boldsymbol{q}}\left(t_{2}\right)\right) \\
\vdots \\
\boldsymbol{\Phi}_{\mathrm{b}}\left(\boldsymbol{q}\left(t_{\mathrm{f}}\right), \dot{\boldsymbol{q}}\left(t_{\mathrm{f}}\right), \ddot{\boldsymbol{q}}\left(t_{\mathrm{f}}\right)\right)
\end{array}\right), \quad \boldsymbol{b}=\left(\begin{array}{c}
\boldsymbol{\tau}_{\mathrm{m}, \mathrm{f}}\left(t_{1}\right) \\
\boldsymbol{\tau}_{\mathrm{m}, \mathrm{f}}\left(t_{2}\right) \\
\vdots \\
\boldsymbol{\tau}_{\mathrm{m}, \mathrm{f}}\left(t_{\mathrm{f}}\right)
\end{array}\right)
$$

where $\tau_{\mathrm{m}, \mathrm{f}}\left(t_{i}\right)$ are friction-corrected torque measurements using prior knowledge of friction parameters $\boldsymbol{d}_{\mathrm{v}, \mathrm{p}}$ and $\boldsymbol{\mu}_{\mathrm{c}, \mathrm{p}}$. The covariance matrix $\boldsymbol{\Sigma}$ is composed of actuator noise variances. Torque measurements $\tau_{\mathrm{m}}$ are determined based on chamber pressures for the hydraulic joints and electric currents for the electromechanic joints. Gear ratios and motor constants are provided by the manufacturer. The joint angle $\boldsymbol{q}$ is measured by position encoders. The resulting parameter vector $\boldsymbol{\beta}_{\mathrm{II}}$ of the sequential method, which is used to parameterize (14), consists of the elements $\boldsymbol{\beta}_{\mathrm{II}}=\left(\boldsymbol{\beta}_{\mathrm{b}} \boldsymbol{d}_{\mathrm{v}, \mathrm{p}} \boldsymbol{\mu}_{\mathrm{c}, \mathrm{p}}\right)^{\mathrm{T}}$.

\footnotetext{
${ }^{1}$ The 70 dynamic parameters ( 10 per joint) are reduced to $n_{\mathrm{b}}=45$ in the minimal regressor form. Additionally, the model has $2 n_{\mathrm{j}}=14$ friction parameters (2 per joint) resulting in $n_{\beta}=59$ parameters overall, see [24].
} 


\section{This is the author's version of an article that has been published in the IROS 2016 proceedings.}

Changes were made to this version by the publisher prior to publication.

The final version of record is available at http://dx.doi.org/10.1109/iros.2016.7759517

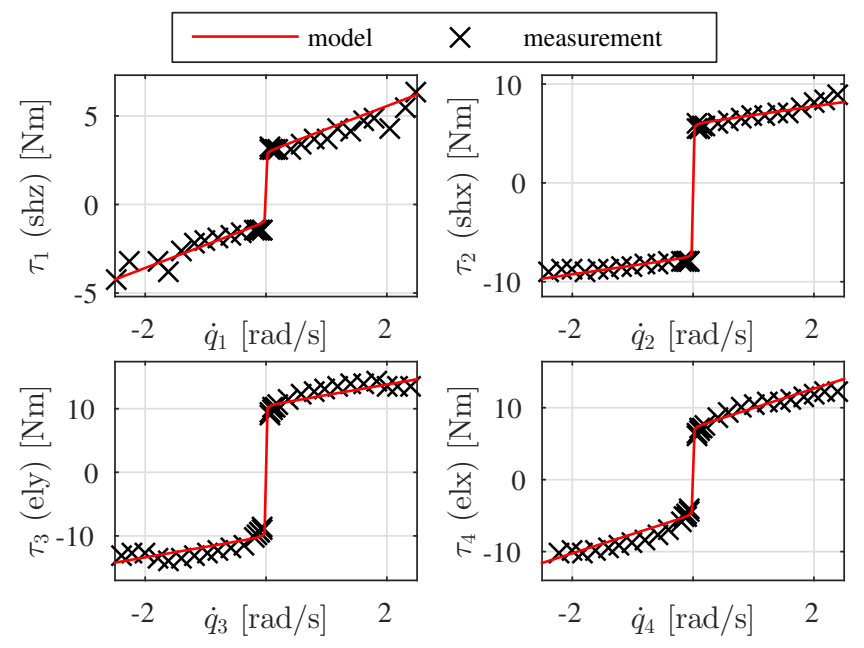

Fig. 4: Viscous and Coulomb friction for the hydraulic joints of the left arm. Each marker represents the mean value of one constant velocity single joint experiment, see Fig. 5. Dynamics effects from $\boldsymbol{\Phi}_{\mathrm{b}}$ were removed and calculated with dynamics parameters $\hat{\boldsymbol{\beta}}_{\mathrm{b}, \mathrm{I}}$ from the combined identification approach [24] and with assumed fixed and upright upper body orientation.
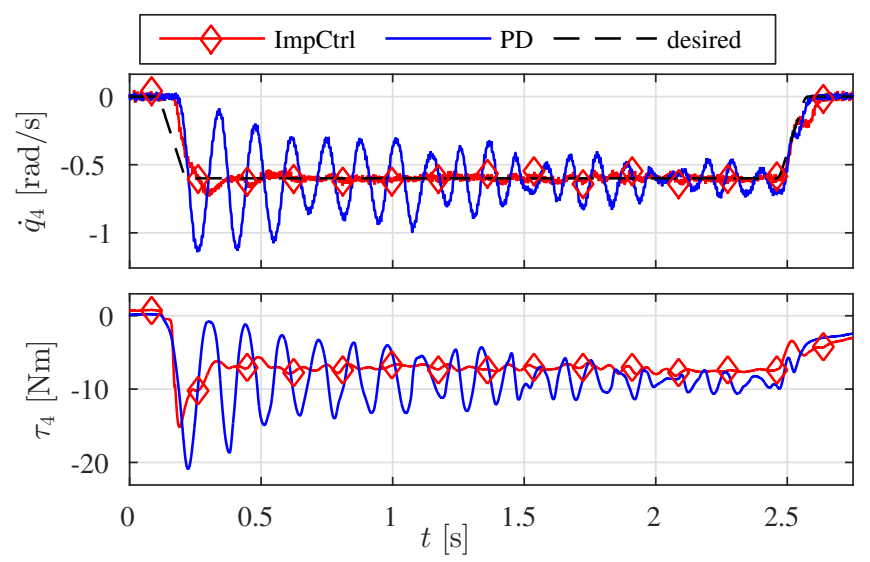

Fig. 5: Single joint friction experiment exemplified for joint 4 reveals improved velocity tracking of the impedance controller compared to PD position control with controller gains from [5], [6].

2) Single Joint Friction Identification: The identification of joint friction parameters $\boldsymbol{d}_{\mathrm{v}, \mathrm{p}}$ and $\boldsymbol{\mu}_{\mathrm{c}, \mathrm{p}}$ is done by running a set of different constant velocities $\dot{q}_{i}$ in positive and negative direction and measuring the resulting torque $\tau_{\mathrm{m}}$ for every joint. Mean velocity and torque are calculated using intervals of constant speed. Figure 4 depicts the joint friction characteristics for the hydraulic joints which show significant Coulomb and viscous friction.

The identification results could be further improved by using the impedance controller to execute the identification trajectory, as it shows significantly improved velocity tracking compared to an extensively tuned PD controller implemented by the manufacturer, see Fig. 5. The identified friction parameters are given in Table I.

3) Results of the Sequential Identification: A comparison between the base parameter vector $\hat{\boldsymbol{\beta}}_{\mathrm{I}}=\left(\hat{\boldsymbol{\beta}}_{\mathrm{b}, \mathrm{I}} \hat{\boldsymbol{d}}_{\mathrm{v}} \hat{\boldsymbol{\mu}}_{\mathrm{C}}\right)^{\mathrm{T}}$, where friction was identified as part of the combined least squares optimization [24], and the base parameter vector $\hat{\boldsymbol{\beta}}_{\text {II }}$ of the sequential method can be found in Fig. 6. Good model consistency is indicated by low distance between
TABLE I: Mean square errors (MSE) for arm identification using different base parameter vectors $\hat{\boldsymbol{\beta}}_{\mathrm{I}}, \hat{\boldsymbol{\beta}}_{\mathrm{II}}$ and data from Fig. 6 and identified friction parameters from single-joint experiments

\begin{tabular}{c|c|c|c|c} 
joint & $\begin{array}{c}\mathrm{MSE}\left(\boldsymbol{\tau}_{\mathrm{m}}-\boldsymbol{\tau}_{\mathrm{I}}\right) \\
{\left[(\mathrm{Nm})^{2}\right]}\end{array}$ & $\begin{array}{c}\mathrm{MSE}\left(\boldsymbol{\tau}_{\mathrm{m}}-\boldsymbol{\tau}_{\mathrm{II}}\right) \\
{\left[(\mathrm{Nm})^{2}\right]}\end{array}$ & $\begin{array}{c}\hat{\mu}_{\mathrm{C}, \mathrm{p}, i} \\
{[\mathrm{Nm}]}\end{array}$ & $\begin{array}{c}\hat{d}_{\mathrm{v}, \mathrm{p}, i} \\
{[\mathrm{Nms} / \mathrm{rad}]}\end{array}$ \\
\hline 1 (shz) & 97.04 & 13.03 & 2.0 & 1.3 \\
2 (shx) & 52.64 & 20.78 & 6.7 & 0.9 \\
3 (ely) & 22.58 & 16.62 & 10.3 & 1.6 \\
4 (elx) & 25.22 & 18.66 & 6.1 & 2.7 \\
\hline 5 (wry) & 2.82 & 6.50 & 0.1 & 0.5 \\
6 (wrx) & 8.11 & 3.46 & 0.1 & 0.2 \\
7 (wry2) & 0.26 & 18.28 & 3.1 & 0.2
\end{tabular}
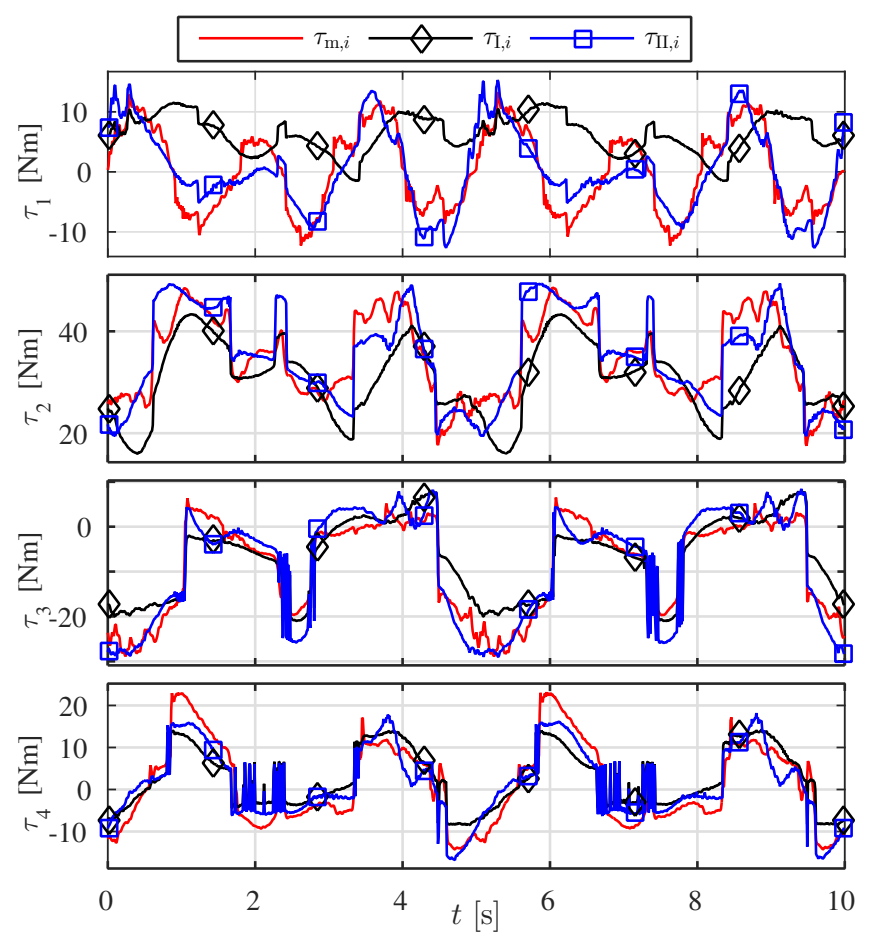

Fig. 6: Measured $\left(\tau_{\mathrm{m}}\right)$ and simulated torques of the left arm joints comparing the sequential method $\tau_{\text {II }}$ with the combined method $\tau_{\mathrm{I}}$.

model and measurement. To avoid the problem of overfitting, the trajectory of this experiment was different from the one used for identification. When using the parameter vector $\hat{\boldsymbol{\beta}}_{\text {II }}$, significantly improved results could be achieved in the hydraulic joints, indicated by the lower mean square error between measured and modeled torques in Table I.

As already mentioned in [24], the electromechanic wrist joints (wry, wrx, wry2) do not seem to be identifiable for the Atlas system without working joint torque sensors. Although friction was identified in single axis experiments, the predicted torques have essentially no correlation with the measured torques. Presumably, this is due to the currentbased torque measurement on actuator side, which decreases the quality of the measured information significantly.

In the next section, the results for the experimental collision handling performance with the Atlas system are outlined.

\section{Collision Detection}

In the following experiments (Sec. III-A and III-B), we used the collision handling (12) and (13) to detect collisions 


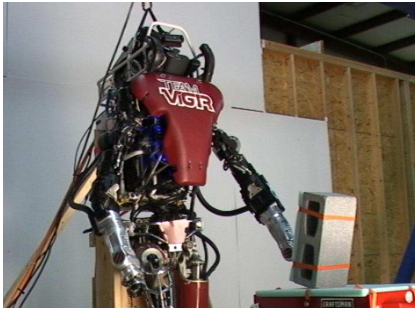

(a) Enabled ("Coll.Det" in Fig. 8)

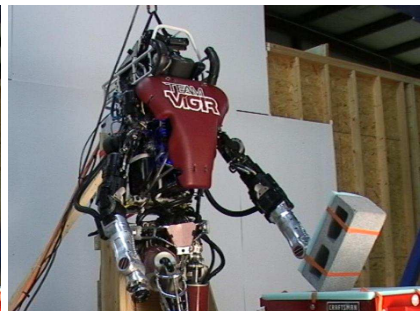

(b) Disabled ("Stiff" in Fig. 8)

Fig. 7: Result after the end-effector collision with and without detection and reaction (snapshot at $t \approx 1.5 \mathrm{~s}$ of Fig. 8 )

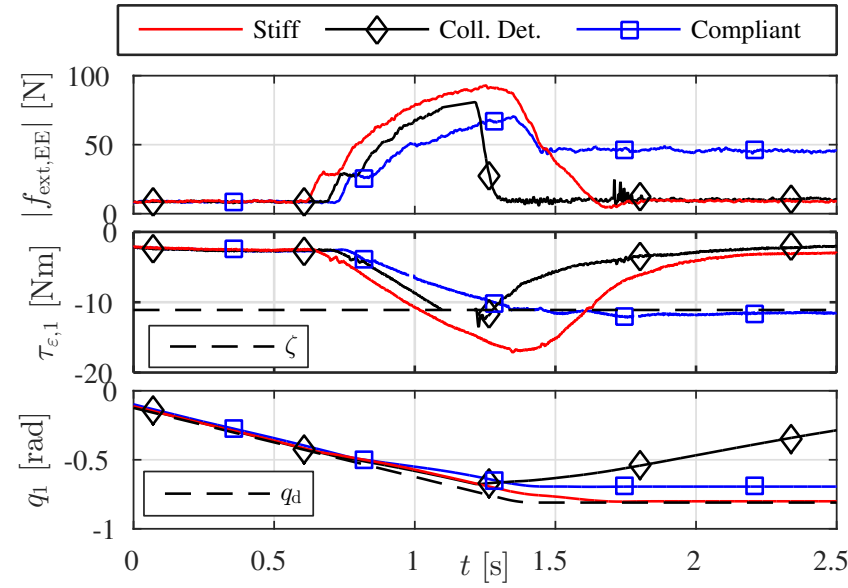

Fig. 8: Absolute external force $\left|\boldsymbol{f}_{\text {ext,EE }}\right|$, observed disturbance torque $\hat{\tau}_{\varepsilon, 1}$ and joint position $q_{1}$ are depicted for the cinderblock collision experiment (see also Fig. 7). For the "Stiff" and "Coll.Det" mode, $k_{i}=300 \mathrm{Nm} / \mathrm{rad}$ and for the "Compliant" mode $k_{i}=100 \mathrm{Nm} / \mathrm{rad}$ were chosen.

with the Atlas robot with a disturbance joint torque threshold $\boldsymbol{\zeta}$ of $11 \mathrm{Nm}$ for each joint. A lower threshold frequently leads to false alarms due to remaining modeling errors (see Fig. 6) and the large friction effects (see Fig. 4). Our model is not able to reliably remove the static friction torque from the observer $\left(\kappa_{\mathrm{f}}=1\right.$ in (6)) due to the indeterminate friction state for low velocities [22]. The friction compensation in (2) currently leads to unwanted oscillations indicating closedloop stability problems originating in the velocity feedback, as described in [6]. Therefore, this feature is disabled in the experiments with $\kappa_{\mathrm{f}}=0$. However, it is possible to reliably detect soft collisions at moderate velocity $(100 \mathrm{~mm} / \mathrm{s})$ in adequate time with the Atlas system. For the collision detection and reaction experiments, the disturbance compensation in (2) was disabled $\left(\kappa_{\varepsilon}=0\right)$. The first joint was selected for evaluation of the experiments, since the collision is detected at this joint first. The other axes show qualitatively the same behavior.

\section{A. End-effector Collision}

The first experiment lets the end-effector collide with a styrofoam-protected cinderblock, see Fig. 7. The protection aimed to protect the robot from damage and has no major influence on the collision detection. The measured data during the three runs with different settings is depicted in Fig. 8. Using a rather stiff joint impedance controller with jointwise $k_{i}=300 \mathrm{Nm} / \mathrm{rad}$ ("Stiff") and deactivated collision handling leads to pushing the cinderblock away and tipping it over till reaching the final goal position, see Fig. 7 (b)

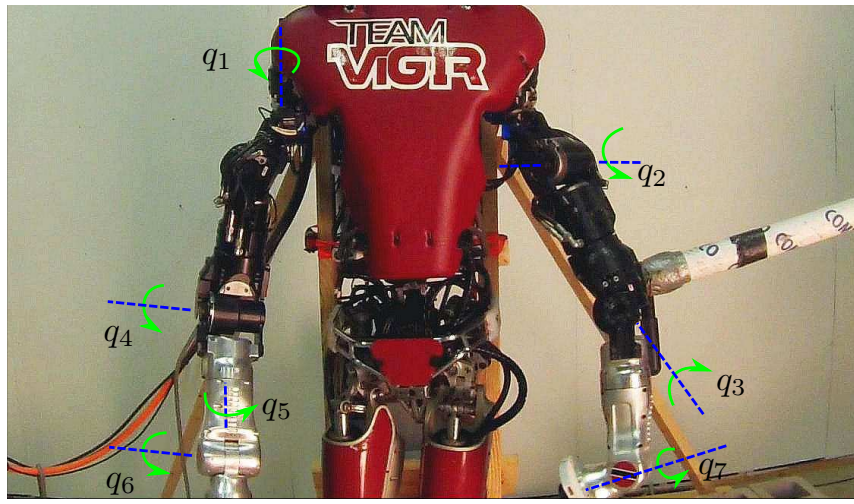

Fig. 9: Scene during the collision from Fig. 10 at $t \approx 5.5 \mathrm{~s}$.
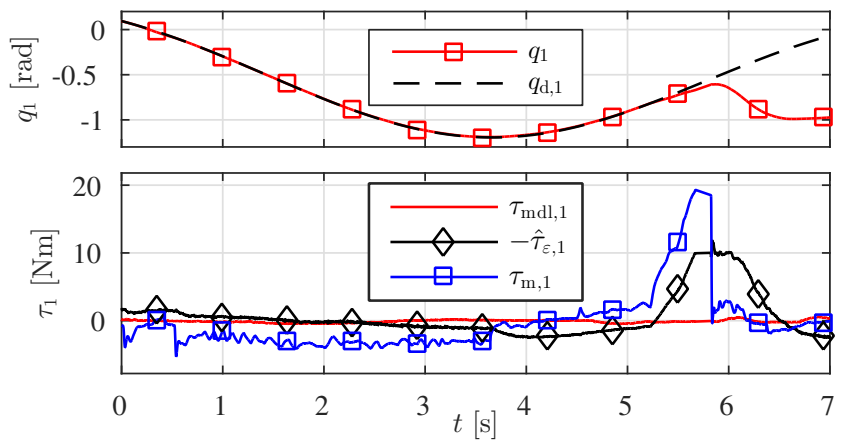

Fig. 10: Joint position $q_{1}$, model torque $\tau_{\mathrm{mdl}, 1}$, estimated disturbance torque $\hat{\tau}_{\varepsilon, 1}$ and motor-side joint torque $\tau_{\mathrm{m}, 1}$ during link collision, see Fig. 9.

and Fig. 8. Reducing the joint stiffness to $k_{i}=100 \mathrm{Nm} / \mathrm{rad}$ ("Compliant") leads to smaller resulting quasi-static contact forces, while the positioning error increases, see Fig. 8.

Using the collision detection and reaction ("Col. Det."), the arm drifts away after the collision has been detected and the contact force decays to zero, see Fig. 7 (a) and Fig. 8. The collision in Fig. 8 is detected at $t=1.1 \mathrm{~s}$, when the disturbance torque line crosses the detection threshold $\zeta$. At this point in time, the maximum contact force of about $69 \mathrm{~N}$ is already reached, which is caused by the relatively slow dynamics of the observer $\left(k_{\mathrm{o}, i}=5 \mathrm{~s}^{-1}\right)$. A larger gain would allow an earlier detection with less reaction force. Using retract reflexes would allow further reduction of contact forces. In turn, larger observer gain causes vibrations when being used as disturbance compensation. To overcome this issue, two observers could be used in parallel: a slower one for compensation and a faster one for collision detection.

\section{B. Link Collision}

The second experiment shows that collisions at the proximal links and not only at the end-effector are detected as well. The arm was pushed at the elbow with a cardboard stick against the moving direction during a joint trajectory, see Fig. 9 (in the depicted situation, the elbow was moving to the right). The movement speed was $\approx 50 \mathrm{~mm} / \mathrm{s}$ at the collision location. Figure 10 depicts the measurement data. The collision at link 4 starts at $t \approx 5.5 \mathrm{~s}$, which one can see from the high motor torque in joint 1 . The detection threshold is reached at $t \approx 5.6 \mathrm{~s}$, leading to the halt in gravity compensation mode. 


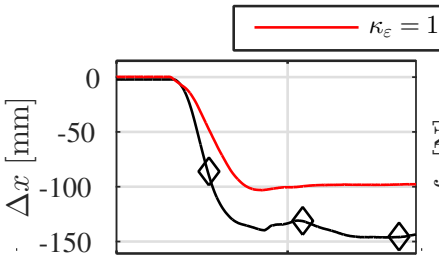

(a) $\quad 0 \quad t[\mathrm{~s}] \quad 2$

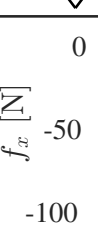

(b)
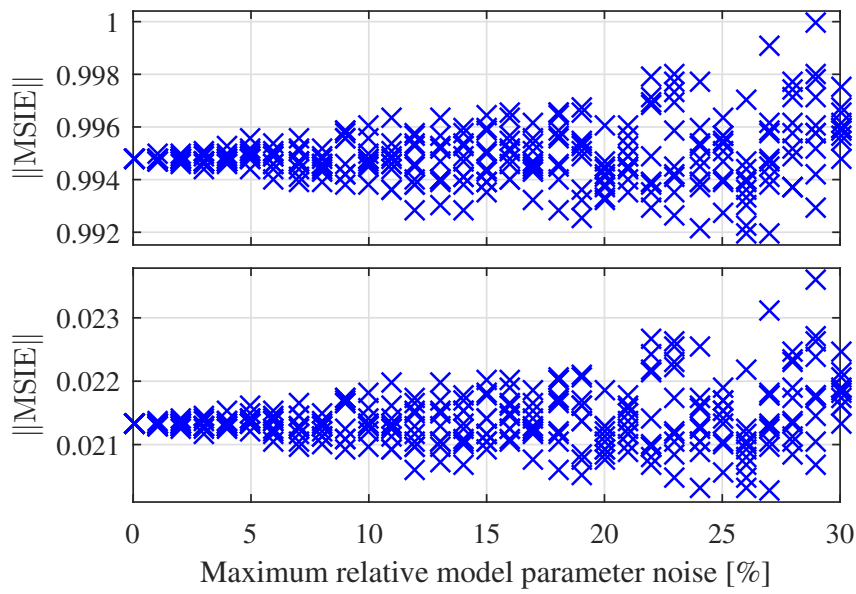

Fig. 13: Simulation results: The experiment of Fig. 12 was done multiple times with different model errors. The normalized mean squared integrated error is plotted over the maximum parameter noise level. The results for $\kappa_{\text {ext }}=0$ and $\kappa_{\text {ext }}=1$ are shown in the top and the bottom plot respectively.

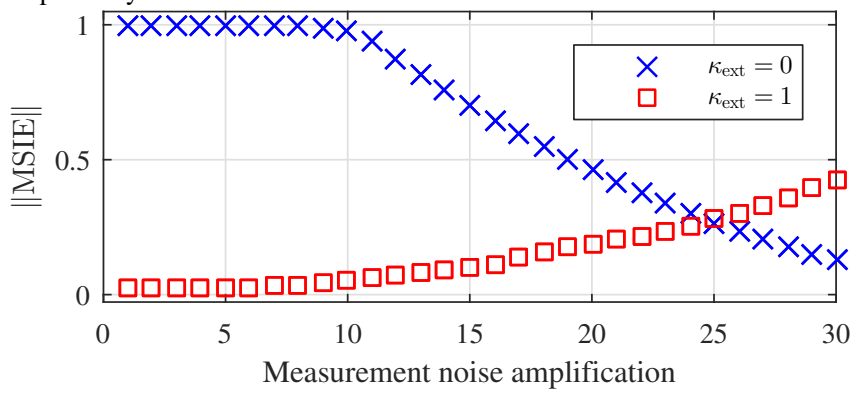

Fig. 14: Simulation results: The experiment from Fig. 12 was done multiple times with different measurement errors. The normalized mean squared error is depicted over the scaling factor applied to all measurement noise levels from the simulation of Fig. 12.

tributed pseudo random numbers between $\pm 10 \%$ as a scaling factor to all masses, inertias, center of mass coordinates and friction coefficients. The sensor noise was (component-wise) uniformly distributed between $\pm 3.4 \cdot 10^{-4} \mathrm{rad}$ for joint position, $\pm 5.5 \cdot 10^{-2} \mathrm{rad} / \mathrm{s}$ for velocity, $\pm 2 \cdot 10^{-4} \mathrm{~m} / \mathrm{s}^{2}$ for the gravity vector, $\pm 7.3 \cdot 10^{-2} \mathrm{Nm}$ for the joint torque and $\pm 2.3 \mathrm{~N}$ for the external forces, respectively. These values were obtained from the measurement data of the hydraulic joints during the starting phase of the experiment in Fig. 11.

For this setup, a step change of the external force acting on the end-effector was performed with different observer settings. The results are shown in Fig. 12. For $\kappa_{\varepsilon}=0$, i.e. applying no disturbance compensation, compliant behavior is achieved. Using the disturbance compensation without EFC $\left(\kappa_{\text {ext }}=0\right)$ leads to a very stiff response according to (10), with $\hat{\tau}_{\varepsilon, 1}$ converging to $\tau_{\text {ext }, 1}$. When selecting $\kappa_{\text {ext }}=1$, the observer torque remains near zero, as it only observes the model errors. In addition, the compliance is the same as without disturbance compensation, i.e. as expected from (11). Therefore, the simulation results imply, that this approach allows to compensate for model errors, while correctly reacting compliant to end-effector contacts at the same time.

3) Robustness of Disturbance Compensation with EFC against Model Errors: Further simulations (as in Fig. 12) 
were performed to investigate the robustness of the above method. First, the influence of model errors is investigated. The model errors were introduced by scaling the masses, the center of gravity positions of the links, the link inertias and the friction parameters by uniformly distributed random factors. The normalized mean squared integrated error (MSIE)

$$
\text { MSIE }=\frac{1}{n_{\mathrm{j}} T_{\mathrm{f}}} \sum_{i=1}^{n_{\mathrm{j}}} \int_{0}^{T_{\mathrm{f}}}\left(q_{i}(t)-q_{i}^{\mathrm{ref}}(t)\right)^{2} \mathrm{~d} t
$$

is depicted over the maximum noise factor for limits from $1 \%$ to $30 \%$ in Fig. 13 for the controller with and without EFC. The norm $\|$ MSIE $\|=$ MSIE/ $\max$ (MSIE) is calculated for Fig. 13 and Fig. 14 separately. The compliant behavior of the controller without disturbance compensation and without model errors or measurement noise was selected as reference $q^{\text {ref }}$. In order to gain comparable results, the model errors were the same in both plots. It can be seen that the influence of the model errors is similar for both cases and the results with EFC remain close to the reference case. The order of magnitude between the results with and without EFC stays the same for all model error levels. Even with an identification error of up to $30 \%$, the controller can cope with the resulting model errors for this quasi-static movement.

4) Robustness of Disturbance Compensation with EFC against Sensor Noise: Figure 14 depicts the influence of measurement noise on controller performance. The measurement noise levels from above were scaled by factors of 1 to 30 and the MSIE is compared for the controller with $\left(\kappa_{\text {ext }}=1\right)$ and without EFC $\left(\kappa_{\text {ext }}=0\right)$. It can be seen that for noise levels amplified up to 10 times, the deviation from the reference behavior does not change noticeably in either case, i.e. the compliance rendering of the controller remains the same despite increased noise.

For larger measurement noise the deviation from the desired behavior becomes smaller for $\kappa_{\text {ext }}=0$ since the limitation of the observer output prevents the compensation of external forces for high measurement noise. This effect even leads to a behavior closer to the desired one with $\kappa_{\text {ext }}=0$ instead of $\kappa_{\text {ext }}=1$ for very high measurement noise (at least 25 times as high as observed in the experiments). In term, Fig. 14 implies that the controller is able to cope with measurement noise about 10 times higher than found in our experimental setup. To cope with larger measurement noise, one would have to use filtering or increase the output limit of the observer.

\section{CONCLusion}

In this paper we designed and implemented a two-step identification method and an adapted generalized momentum observer for articulated robots. We applied both methods to the arms of the humanoid robot Atlas. The two-step identification significantly improves the identification results in the presence of high friction in the examined scenario compared to our previous work. The adapted observer enables precise and compliant manipulation. In addition, friction models can be incorporated to further improve the observer performance.
The proposed solution is able to detect collisions along the entire robot structure. Overall, this provides key features to safely operate in unknown environments and is a first step towards the safe cooperation of (partly) hydraulically driven humanoids with humans.

\section{REFERENCES}

[1] A. De Luca, A. Albu-Schäffer, S. Haddadin, and G. Hirzinger, "Collision detection and safe reaction with the DLR-III lightweight manipulator arm," in IROS, 2006.

[2] S. Haddadin, Towards Safe Robots, pp. 34-37. No. 90 in Springer Tracts in Advanced Robotics, Springer Berlin Heidelberg, 2014.

[3] A. Bicchi and G. Tonietti, "Dealing with the safety-performance tradeoff in robot arm design and control: Fast and "soft-arm" tactics," IEEE Robotics and Automation Magazine, pp. 22-33, June 2004.

[4] A. De Luca and F. Flacco, "Integrated control for pHRI: Collision avoidance, detection, reaction and collaboration," in BioRob, 2012.

[5] S. Kohlbrecher et al., "Human-robot teaming for rescue missions: Team ViGIR's approach to the 2013 DARPA robotics challenge trials," Journal of Field Robotics, vol. 32, no. 3, pp. 352-377, 2015.

[6] D. Conner et al., "Team ViGIR (DARPA final report, accession number ADA623035)," tech. rep., Defense Technical Information Center Document, 2015.

[7] G. Pratt and M. Williamson, "Series elastic actuators," IROS, 1995.

[8] A. Bicchi and G. Tonietti, "Design, Realization and Control of Soft Robot Arms for Intrinsically Safe Interaction with Humans," In Proc. IARP/RAS Workshop on Technical Challenges for Dependable Robots in Human Environments, pp. 79-87, 2002.

[9] N. Hogan, "Impedance control: An approach to manipulation, part I - theory," ASME Journal of Dynamic Systems, Measurement, and Control, vol. 107, pp. 1-7, 1985.

[10] A. Albu-Schäffer, C. Ott, and G. Hirzinger, "A unified passivitybased control framework for position, torque and impedance control of flexible joint robots," IJRR, vol. 26, no. 1, pp. 23-39, 2007.

[11] C. Ott, "Cartesian impedance control of redundant and flexible-joint robots," Springer Tracts in Advanced Robotics, 2008.

[12] S.-H. Hyon, J. Hale, and G. Cheng, "Full-body compliant humanhumanoid interaction: Balancing in the presence of unknown external forces," IEEE Trans. Robot., vol. 23, pp. 884-898, Oct 2007.

[13] A. Herzog, L. Righetti, F. Grimminger, P. Pastor, and S. Schaal, "Balancing experiments on a torque-controlled humanoid with hierarchical inverse dynamics," in ICRA, 2014.

[14] S. Mason, L. Righetti, and S. Schaal, "Full dynamics LQR control of a humanoid robot: An experimental study on balancing and squatting," in Humanoids, 2014.

[15] K.-H. Lee and W. Newman, "Natural admittance control of an electrohydraulic humanoid robot," in ROBIO, 2014.

[16] Y. Oh, W. K. Chung, and I. H. Suh, "Disturbance observer based robust impedance control of redundant manipulators," IROS, 1999.

[17] A. Nikoobin and R. Haghighi, "Lyapunov-based nonlinear disturbance observer for serial n-link robot manipulators," Journal of Intelligent and Robotic Systems, vol. 55, pp. 135-153, Dec 2008.

[18] A. de Luca and R. Mattone, "Sensorless robot collision detection and hybrid force/motion control," ICRA, 2005.

[19] V. Sotoudehnejad and M. R. Kermani, "Velocity-based variable thresholds for improving collision detection in manipulators," in ICRA, 2014.

[20] C. Ott, B. Henze, and D. Lee, "Kinesthetic teaching of humanoid motion based on whole-body compliance control with interaction-

aware balancing," in IROS, 2013 .
[21] S. Golz, C. Osendorfer, and S. Haddadin, "Using tactile sensation for learning contact knowledge: Discriminate collision from physical interaction," ICRA, 2015.

[22] C. Bidard, C. Libersa, D. Arhur, Y. Measson, J.-P. Friconneau, and J.-D. Palmer, "Dynamic identification of the hydraulic maestro manipulator-relevance for monitoring," Fusion Eng. and Design, 2005.

[23] B.-J. Jung, H. R. Choi, J. C. Koo, and H. Moon, "Collision detection using band designed disturbance observer," in IEEE CASE, 2012.

[24] M. Schappler, J. Vorndamme, A. Tödtheide, D. Conner, O. von Stryk, and S. Haddadin, "Modeling, identification and impedance control of the Atlas arms," in Humanoids, 2015.

[25] L. Le Tien, A. Albu-Schäffer, A. De Luca, and G. Hirzinger, "Friction observer and compensation for control of robots with joint torque measurement," in IROS, 2008.

[26] A. De Luca and R. Mattone, "Actuator failure detection and isolation using generalized momenta," in ICRA, 2003.

[27] A. De Luca and R. Mattone, "An adapt-and-detect actuator FDI scheme for robot manipulators," in ICRA, 2004.

[28] K.-J. Park, "Fourier-based optimal excitation trajectories for the dynamic identification of robots," Robotica, 2006. 